\title{
Verrucous DLE: A rare presentation misdiagnosed as plantar warts
}

\author{
Authors \\ Dr Ritu Rani ${ }^{1}$, Dr Reena K Sharma ${ }^{2}$, Dr Mudita Gupta ${ }^{3 *}$ \\ ${ }^{1}$ Dermatologist, Civil Hospital Kangra,H.P. \\ ${ }^{2}$ Dermatologist, Civil Hospital Kangra \\ ${ }^{3}$ Assistant Professor, Department of Dermatology, IGMC, Shimla \\ *Corresponding Author \\ Dr Mudita Gupta \\ Assistant Professor, Department of Dermatology, IGMC, Shimla, India
}

\begin{abstract}
Verrucous DLE (discoid lupus erythematosus) is a rare variant of cutaneous lupus erythematosus which can easily be misdiagnosed as verruca. The morphologic presentation of this rare disease should be kept in mind and should not be missed because of its rarity. A 45 year female with multiple verrucous lesions who was being treated as case of cutaneous warts was diagnosed as verrucous DLE and responded remarkably with hydroxychloroquine.

Keywords: verrucous, DLE, warts, atrophic plaques.
\end{abstract}

\section{Introduction}

Cutaneous lupus erythematosus can present as classical discoid lesion (DLE) or papulosquamous or annular lesions as seen in subacute cutaneous lupus erythematosus. ${ }^{[1]}$ Verrucous or hypertrohic DLE is a rare variant that can be misdiagnosed with conditions like verruca or squamous cell carcinoma. We herein describe a rare presentation of verrucous DLE in a 45 year female who was being treated as cutaneous warts till date.

\section{Case Report}

A 45 year female presented with multiple skin lesions over face, ear, lips and soles for last 3 years. On clinical examination the lesions on face and ears were well defined, atrophic, scaly plaques with hyperpigmented border while the lesions on soles were large, well defined hyperpigmented, hypertrophic verrucous plaques of size ranging from $3 \times 2 \mathrm{~cm}$ approximately. The patient did not have any history of photosentivity, oral lesions or joint symptoms. She had multiple visits at some private institution where she was being treated as a case of cutaneous warts for a long time but no improvement was seen during the course of treatment. The patient's complete hemogram, urine routine examination, liver, and renal function tests were normal. Viral markers were negative. ANA profile was also negative. Our patient did not qualify for the diagnosis of SLE. Histopathogical examination showed irregular epidermal hyperplasia with hyperkeratosis, acanthosis, vacoular basal cell degeneration and peri-appendegeal and lymphohistiocytic infiltrate at dermoepidermal interface. Based on history, clinical examination 
and histopathology we kept the diagnosis of verrucous DLE and started patient on hydroxychloroquine $200 \mathrm{mg}$ twice a day along with intralesional triamcinolone $40 \mathrm{mg} / \mathrm{ml}$ repeated every 3 weeks. Follow up at 6 and 10 weeks was done with remarkable improvement in the lesions.

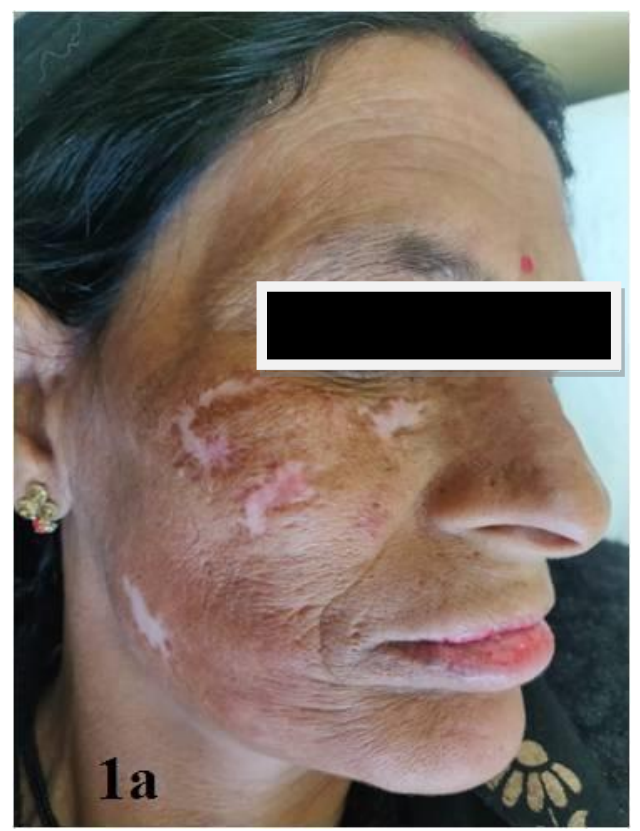

Fig 1a Depigmented, Atrophic Plaques of variable size with hyperpigmented border over face and lips.

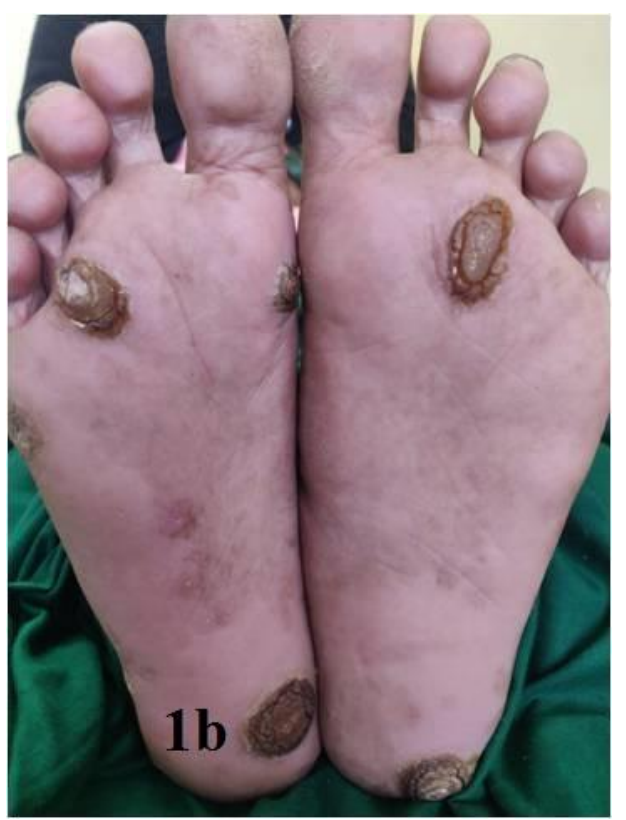

Fig 1b. Hyperkeratotic verrucous plaques over plantar aspect of bilateral feet.

\section{Discussion}

Verrucous DLE was first described by Behçet in 1940 as lupus erythematosus hypertrophicus et profundus. ${ }^{[2]}$ Clinically it is characterized by hypertrophic verrucous plaques with minimal scaling on forearms, face, upper trunk, palms and soles. Other morphological presentations of the disease include violaceous papules, blackish hyperkeratotic ulcers, ${ }^{[3]}$ depigmented atrophic plaques, ${ }^{[4]}$ and keratoacanthoma like papulonodular verrucous lesions. ${ }^{[5]}$ The lesions may simulate hypertrophic lichen planus, verrucae, keratoacanthoma or squamous cell carcinoma. ${ }^{[6]}$ In contrast, classical lesions of DLE present with plaques with atrophy, follicular plugging, and adherent scales.

The Chronic nature, treatment refractory lesions and potential to develop malignancy are causes of concern in verrucous DLE. Squamous cell carcinoma may develop on long standing persistent hypertrophic lupus erythematosus lesions. Regular follow up has to be done in such patients. Various treatment modalities for verrucous DLE include hydroxychloroquine, intralesional triamcinolone, ${ }^{[6]}$ isotretinoin, thalidomide, ${ }^{[7,8]}$ and acitretin. ${ }^{[9]}$

We report this case to highlight the diagnostic dilemma posed by this rare disease. It was misdiagnosed as cutaneous warts and was being treated for warts till date. The clinical presentations of the rare variants of cutaneous lupus should be taken into consideration while approaching the diagnosis.

\section{Conclusion}

The rare variants cutaneous lupus erythematosus like verrucous DLE should be taken into account while approaching the verrucous lesions along with its close differentials like warts that may cause dignostic dilemma.

\section{References}

1. Tebbe B. Clinical course and prognosis of cutaneous lupus erythematosus. Clin Dermatol 2004;22:121- 4. 
2. Bechet PE. Lupus erythematosus hypertrophicus et profundus. Arch Dermatol Syph 1942;45:33-9.

3. Otani A. Lupus erythematosus hypertrophicus et profundus. $\mathrm{Br} \quad \mathrm{J}$ Dermatol 1977;96:75-8.

4. Vinciullo C. Hypertrophic lupus erythematosus: Differentiation from squamous cell carcinoma. Australas J Dermatol 1986;27:76-8 2.

5. Santa Cruz DJ, Uitto J, Eisen AZ, Prioleau PG. Verrucous lupus erythematosus: Ultrastructural studies on a distinct variant of chronic discoid lupus erythematosus. $\mathbf{J}$ Am Acad Dermatol 1983;9:82-90.

6. Thappa DM, Venkateswaran S, Reddy BS, Garg BR. Verrucous cutaneous lupus erythematosus. Indian J Dermatol Venereol Leprol 1995;61:36- 7.

7. Georgala S, Katoulis AC, Hasapi V, Koumantaki- Mathioudaki E. Thalidomide treatment for hypertrophic lupus erythematosus. Clin Exp Dermatol 1998;23:141.

8. Gambini D, Carrera C, Passoni E, Muratori S, Berti E, Caputo R. Thalidomide treatment for hypertrophic cutaneous lupus erythematosus. J Dermatolog Treat 2004;15:365- 71.

9. Al- Mutairi N, Rijhwani M, Nour- Eldin O. Hypertrophic lupus erythematosus treated successfully with acitretin as monotherapy. J Dermatol 2005;32:482- 6 . 